\title{
激光微织构泡沫铜的氧化铜纳米线制备及其光催 化性能研究
}

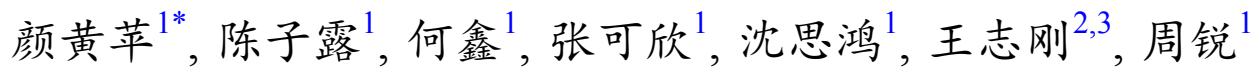

1. 厦门大学航空航天学院, 厦门 361000 ;

2. 武汉科技大学冶金装备及其控制教育部重点实验室, 武汉 430081;

3. 武汉科技大学机械传动与制造工程湖北省重点实验室, 武汉 430081

*联系人, E-mail: hpyan@xmu.edu.cn

收稿日期: 2019-06-22; 接受日期: 2019-08-13; 网络出版日期: 2019-11-20

武汉科技大学机械传动与制造工程湖北省重点实验室开放基金(编号：2017A04)和福建省激光精密加工工程技术研究中心开放课题(编号: 2018JKA006)资助项目

摘要本文采用激光织构与热氧化法相结合, 以泡沫铜为基底制备具有微阵列结构的氧化铜纳米线( CuO NWs). 采用扫描电子显微镜(SEM)、X射线能谱仪(EDS)和X射线衍射仪(XRD)对样品表面形貌及成分进行表征，讨论激 光织构表面微结构对氧化铜纳米线生长的促进作用，设计不同的加工参数研究激光织构对氧化铜纳米线生长的 影响, 并通过光催化降解甲基橙溶液考察了 $\mathrm{CuO} \mathrm{NWs}$ 的光催化性能. 实验结果表明, 经过激光织构的泡沫铜能增 大纳米线的生长面积, 提高样品表面氧元素的含量, 有效地促进生长过程中氧化铜纳米线长度和密度的提高. 不 同的激光参数对纳米线的生长产生一定影响，具有良好的调控作用。 $\mathrm{CuO} \mathrm{NWs}$ 光催化降解甲基橙的降解率可达 97\%, 循环测试5次后的降解率仍可保持在 $96 \%$, 可见其稳定性良好, 而且其片状结构更易回收, 重复利用率高, 具 有优异的光催化性能.

关键词泡沫铜, 氧化铜纳米线, 激光织构, 光催化降解

PACS: 81.05.Rm, 61.46.-w, 42.62.Cf, 81.16.Hc

\section{1 引言}

印染纺织、包装加工、皮革制造等工业排放大量 有机染料废水，未经处理达标的废水流入江河湖泊会 造成污染，甚至在部分废水中含有苯环、胺基、偶氮 基团等致癌物质，对环境及生态产生不可逆转的伤 害 ${ }^{[1,2]}$. 常用的废水处理方法包括物理吸附法 ${ }^{[3]}$ 、电化
学法 ${ }^{[4]}$ 、膜生物处理技术法 ${ }^{[5]}$ 等, 然而都存在能耗 大、成本较高以及使用寿命较短等问题. 光敏半导体 在太阳光的照射下激发出的高活性自由基，可有效降 解有机染料且无二次污染，这种方法逐渐成为处理有 机染料废水的主要趋势. 其中，纳米结构的金属氧化 物半导体材料具有独特的物理和化学性能, 比表面积 更大，反应活性更高，在光催化降解有机染料中具有

引用格式: 颜黄苹, 陈子露, 何釒金, 等. 激光微织构泡沫铜的氧化铜纳米线制备及其光催化性能研究. 中国科学: 物理学 力学 天文学, $2020,50: 034209$ Yan H P, Chen Z L, He X, et al. Fabrication and photocalytic performance of CuO nanowires on copper foam based on laser texturing (in Chinese). Sci Sin-Phys Mech Astron, 2020, 50: 034209, doi: 10.1360/SSPMA-2019-0239 
很大的应用潜力, 如介孔 $\mathrm{TiO}_{2}$ 纳米管 ${ }^{[6]} 、 \mathrm{CdS} / \mathrm{TiO}_{2}$ 纳 米粒子 ${ }^{[7]}, \mathrm{CuS} / \gamma-\mathrm{Fe}_{2} \mathrm{O}_{3}{ }^{[8]} 、 \mathrm{SnO}_{2}-\mathrm{TiO}_{2}$ 核-壳纳米 线 ${ }^{[9]}$ 等.

氧化铜作为一种典型 $p$ 型半导体材料, 禁带宽度为 $1.2-2.0 \mathrm{eV}^{[10]}$, 具有光响应范围较大、无毒性、成本低 等显著优点, 在光催化领域中受到越来越多的关注. 目 前，研究者已通过不同的方法获得多种氧化铜纳米结 构, 包括纳米线 ${ }^{[11]}$ 、纳米球 ${ }^{[12]}$ 和纳米片 ${ }^{[13]}$ 等, 以此增 强氧化铜的光催化性能. 常规的制备方法包括溶胶-凝 胶法 ${ }^{[14]}$ 、水热法 ${ }^{[15]}$ 、共沉积法 ${ }^{[16]}$ 和模板法 ${ }^{[17]}$ 等. 然 而, 大部分由化学法制备的光催化纳米材料由于其形 态特点，存在着难以回收、重复利用率低、易造成二 次污染等问题. 热氧化法是一种步骤简单、成本较低 且可大批量制备氧化铜纳米线的方法 ${ }^{[18]}$. 通过控制氧 化时间和氧化温度可以使纳米线直接生长在铜基底 上. 泡沫铜作为一种新型功能材料, 其多级多孔结构具 有更大比表面积且有利于空气通过，在光催化领域中 有极大的应用价值. 于晶晶等人 ${ }^{[19]}$ 采用一步热氧化法, 在泡沫铜上制备氧化铜纳米线 $(\mathrm{CuO} \mathrm{NWs})$, 光催化降 解甲基橙的降解率可达 $87.2 \%$. 但该方法制备的纳米 线膜仍存在易脱落、出现裂纹等问题，对降解效率及 循环利用率存在不利影响.

激光织构金属表面被认为是一种高效、精确地制 备微纳功能化表面的方法 ${ }^{[20,21]}$. 针对传统热氧化法生 长 $\mathrm{CuO} \mathrm{NWs}$ 存在的不足, 本文以泡沫铜为基底, 采用 激光微织构与热氧化相结合的方法制备 $\mathrm{CuO} \mathrm{NWs}$ 光 催化剂. 此方法操作简单、成本低廉，其自支撑的微 纳米结构不仅能解决传统颗粒光催化剂难以回收、易 二次污染等问题，而且可有效增大比表面积并增强纳 米线的附着力, 提高光催化剂的催化效率和重复利 用率.

\section{2 材料与方法}

\section{1 实验材料与分析设备}

样品使用 $25 \mathrm{~mm} \times 25 \mathrm{~mm} \times 0.5 \mathrm{~mm}$ 的泡沫铜(昆山 广嘉源新材料有限公司, 中国); 实验试剂包括盐酸 $(36 \%)$ 、过氧化氢(30\%)、无水乙醇(分析纯，西陇科学 股份有限公司, 中国); 甲基橙(天津市致远化学试剂有 限公司, 中国); 实验用水为去离子水. 采用扫描电子显 微镜(SEM, ZEISS SUPRA 55, 德国)对样品的表面形
貌进行表征; 采用X射线能谱仪(EDS，ZEISS Xradia 800 Ultra, 德国)对样品表面的化学元素成分及含量进 行分析; 采用X射线衍射仪(XRD, XRD-7000, 岛津, 日 本)对样品进行物相分析; 采用紫外-可见分光光度计 (UV-Vis, Genesys 10S, 赛默飞, 美国)测量溶液的吸 光度.

\section{2 氧化铜纳米线的制备}

首先，将泡沫铜经盐酸、无水乙醇及去离子水洗 净, 用氮气吹干后, 使用纳秒光纤激光器 (波长 $1064 \mathrm{~nm}$ ，重复频率 $20 \mathrm{kHz}$ ，脉宽200 ns)对泡沫铜表面 以“井字型”扫描图案进行激光微织构. 激光光斑聚焦 直径为 $35 \mu \mathrm{m}$, 输出功率为 $20 \mathrm{~W}$, 扫描次数分别为 5 次、 10 次和 20 次, 激光扫描间距分别为 $0.1,0.3$ 和 $0.5 \mathrm{~mm}$. 将激光加工过的泡沫铜放置于加热板上, 在 $500^{\circ} \mathrm{C}$ 的条件下进行热氧化. 加热 $5 \mathrm{~h}$ 后, 以 $5^{\circ} \mathrm{C} / \mathrm{min}$ 的 速率退火降至室温, 得到具有微纳米结构的 $\mathrm{CuO} \mathrm{NWs.}$ $\mathrm{CuO} \mathrm{NWs}$ 制备过程如图1所示.

\section{3 光催化性能测试}

配制 $40 \mathrm{mg} / \mathrm{L}$ 的甲基橙溶液, 将制备好的样品作为 催化剂放入如图2所示的光催化反应装置内. 光催化装 置的主体部分是反应管, 为避免自然光的干扰, 在反应 管的外侧包覆铝䈃纸. 管腔内放置灯管与防水罩, 管壁 通有循环水, 使反应温度始终保持在 $23^{\circ} \mathrm{C}$.

经过 $1 \mathrm{~h}$ 的暗处理后达到吸附平衡, 然后在紫外灯 $(10 \mathrm{~W} ， 254 \mathrm{~nm}$ )照射下，每隔 $5 \mathrm{~min}$ 取样，在 $1 \mathrm{~h}$ 后每隔 $10 \mathrm{~min}$ 取样, 离心后用分光光度计在波长为 $465 \mathrm{~nm}$ 处 测量其吸光度, 记录数值. 设置对照实验, 分别为无催 化剂组、直接氧化组以及激光处理氧化组, 并进行平 行实验.

\section{3 实验结果和分析}

\section{1 氧化铜纳米线的表面形貌}

图3为样品表面形貌的SEM图像. 图3(a)和(d)为原 始泡沫铜样品表面形貌图. 由(a)可看出泡沫铜的多层 网状结构, 由(d)可见样品存在纹路, 表面光滑. 图3(b) 和(e)为直接热氧化泡沫铜的表面形貌图. 由(b)可以看 出泡沫铜的骨架仍保留完整, 其表面在氧化后出现“毛 线状”的纳米线, 由(e)可以更清楚地看到 $\mathrm{CuO} N \mathrm{NW}$ 虽 

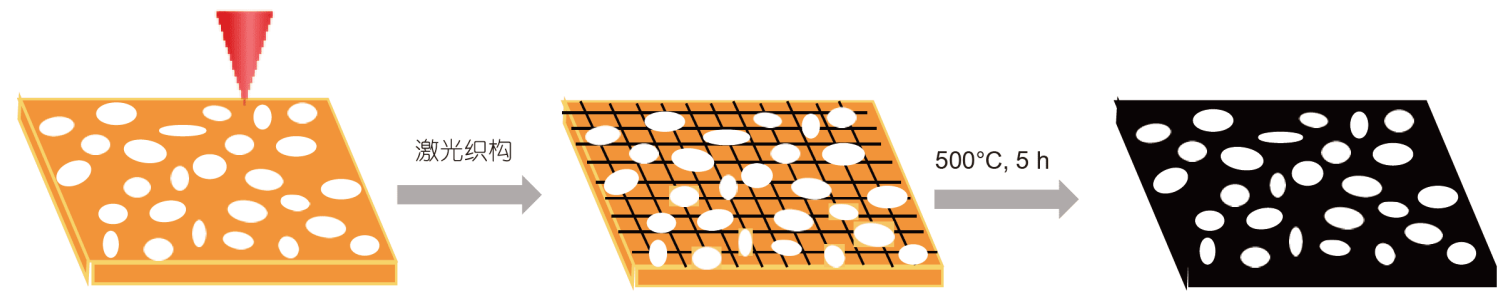

图 1 (网络版彩图) $\mathrm{CuO} \mathrm{NWs}$ 的制备方法示意图

Figure 1 (Color online) Schematic diagrams of fabricating CuO NWs.

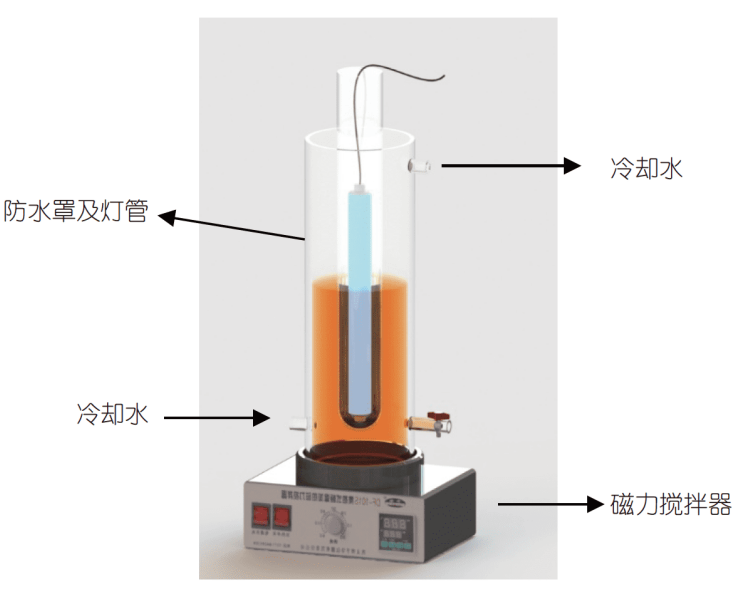

图 2 (网络版彩图)光催化反应装置图

Figure 2 (Color online) Diagram of photocatalytic reactor.

有生长, 但纳米线生长方向无序交错, 呈杂草状, 表面 较为稀疏倒伏且分布不均匀, 长度大概为 $0.5-2 \mu \mathrm{m}$, 存 在部分脱落的情况. 图3(c)和(f)为经过激光预处理后
热氧化的 $\mathrm{CuO} \mathrm{NWs}$ 表面形貌图. (c)表示在激光扫描间 距为 $0.1 \mathrm{~mm}$, 扫描次数 20 次的条件下, 将泡沫铜加工出 “井字形”微阵列结构. 由(f)可清晰地看出, $\mathrm{CuONWs}$ 分 布均匀且生长致密，纳米线基本保持直立生长而不交 错, 长度大概为 $4-10 \mu \mathrm{m}$, 较(d)明显更长. 通过图3的对 比分析可知，使用激光微织构对泡沫铜表面进行前期 处理可直接影响热氧化过程中 $\mathrm{CuO} \mathrm{NWs}$ 的生长, 纳米 线生长密度更高、长度更长且比表面积显著增加.

图4为不同扫描次数下的氧化铜纳米线表面形貌 图. 由图4(a)-(c) 可明显看出, 当扫描次数为 5 次时, 激 光织构的效果不是很明显, 泡沫铜的骨架基本保留; 扫 描次数为 10 次时, 泡沫铜的骨架轮廓模糊, 已基本断 裂, 激光织构的微阵列结构较为清晰; 当扫描次数为 20次时，泡沫铜表面及下层的原始骨架已消失，激光 烧蚀较严重, 激光织构的微阵列结构代替其原始孔洞 结构. 根据图4(d)-(f)的表面形貌图分析可得，仅激光 扫描5次后生长的 $\mathrm{CuO} \mathrm{NWs}$ 虽也能基本保持直立生长,
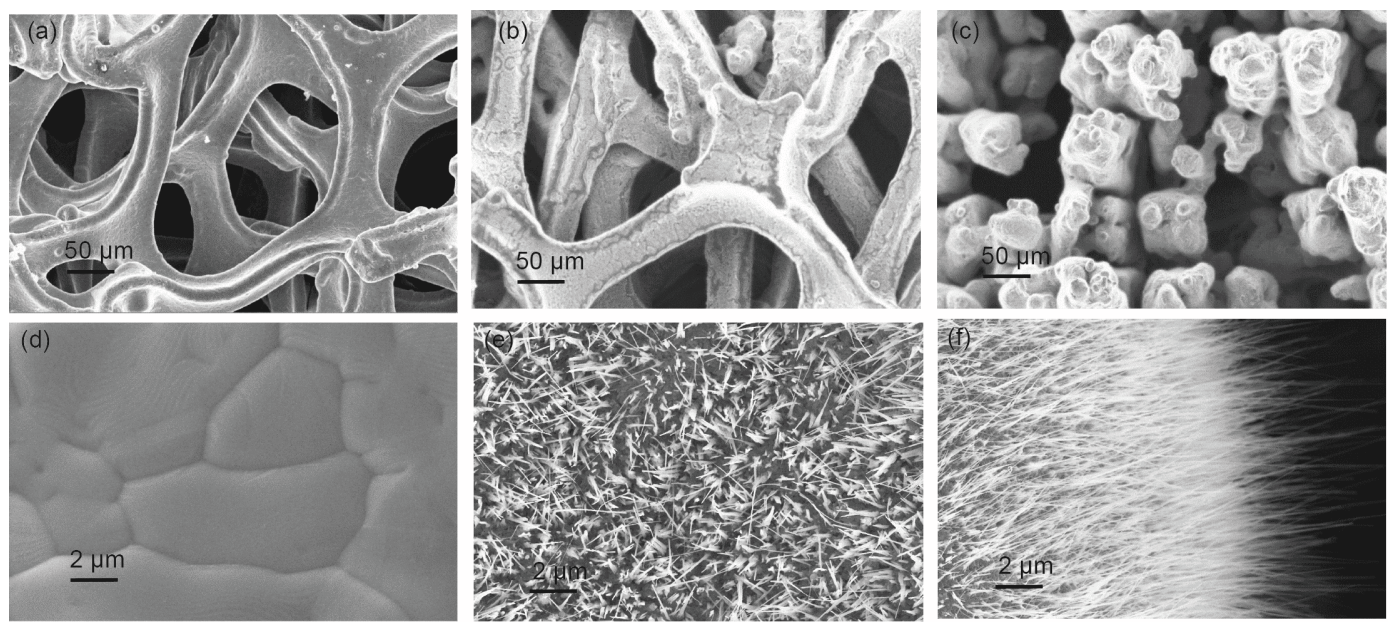

图 3 氧化铜纳米线的表面形貌图. (a), (d) 原始样品组; (b), (e) 直接氧化组; (c), (f) 经过激光预处理组

Figure 3 SEM images of the $\mathrm{CuO}$ nanowire surfaces. (a), (d) Original sample; (b), (e) $\mathrm{CuO}$ NWs prepared by direct thermal oxidation; (c), (f) CuO NWs prepared by laser texturing and thermal oxidation. 
但较为稀疏，纳米线长度在 $1-5 \mu \mathrm{m}$; 而激光扫描 10 次 和 20 次得到的氧化铜纳米线不仅保持直立, 生长也更 加茂盛, 长度在4-10 $\mu \mathrm{m}$.

图5为不同扫描间距下的表面形貌图. 由图5(a)和 (d)可以看出，在扫描间距为 $0.1 \mathrm{~mm}$ 时，激光织构形成 的微阵列结构完全代替了泡沫铜的原始孔洞结构. 无 论是上表面或激光织构形成的凹槽侧面, 纳米线保持 直立生长且分布均匀, 长度在4-10 $\mu \mathrm{m}$. 由图 $5(\mathrm{~b})$ 可清 晰看出, 在扫描间距为 $0.3 \mathrm{~mm}$ 时, 泡沫铜表面的微阵列
结构以及部分泡沫铜的完整孔洞结构, 由图5(e)对比 可得，上表面未被烧蚀和被烧蚀区域的纳米线生长差 异明显. 未被烧蚀的表面, 纳米线生长稀疏且分布不 均, 呈倒伏状, 长度基本在 $0.5-2 \mu \mathrm{m}$; 而激光扫描路径 经过的区域, 纳米线密度有明显提高, 长度在 $4-10 \mu \mathrm{m}$, 且保持直立生长. 图 $5(\mathrm{c})$ 和(f)表示扫描间距为 $0.5 \mathrm{~mm}$ 时的泡沫铜形貌图, 泡沫铜的孔洞只有激光扫描过的 区域存在被烧蚀破坏的痕迹, 纳米线的生长情况与扫 描间距为 $0.3 \mathrm{~mm}$ 的情况类似, 未烧蚀过的区域所生长
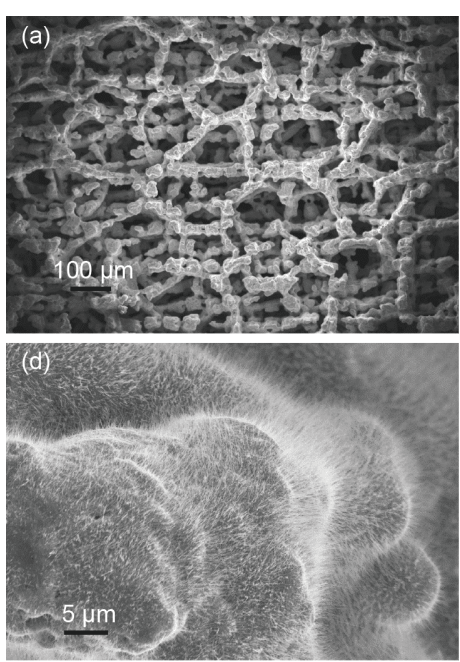
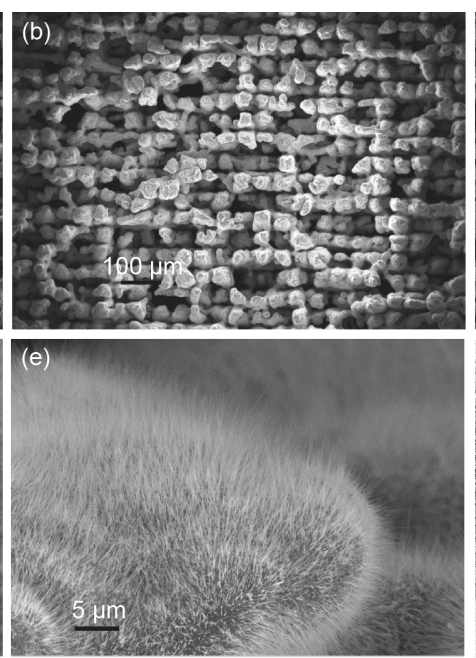
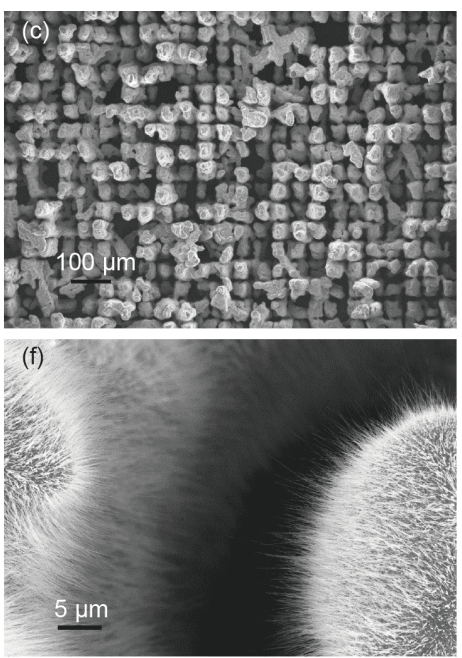

图 4 不同扫描次数下的氧化铜纳米线形貌图. (a), (d) 5次; (b), (e) 10次; (c), (f) 20次

Figure 4 SEM images of the $\mathrm{CuO}$ nanowire surfaces with different scan times. (a), (d) 5 times; (b), (e) 10 times; (c), (f) 20 times.
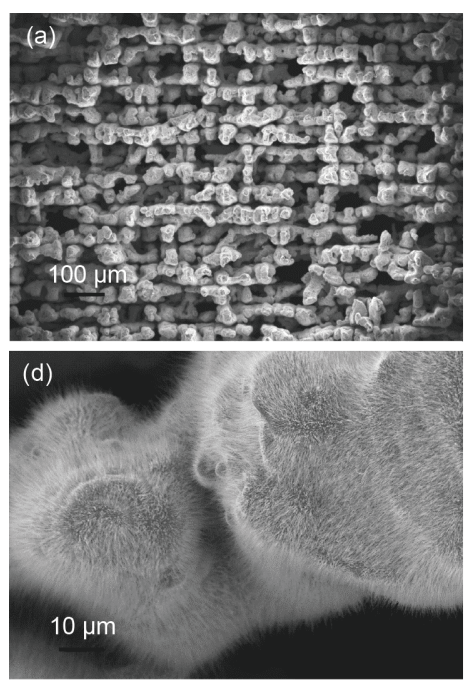
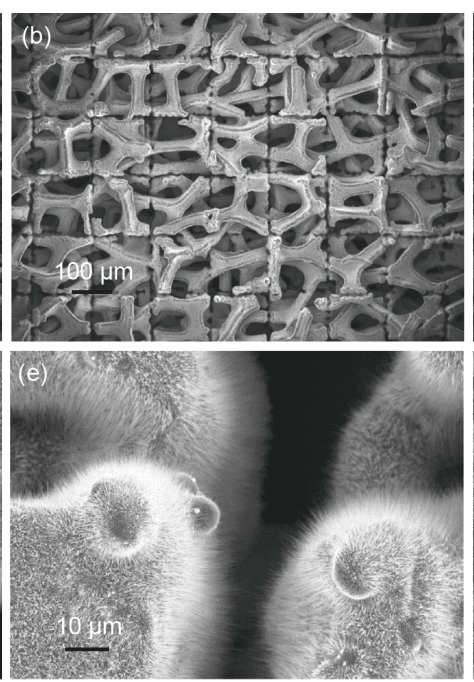
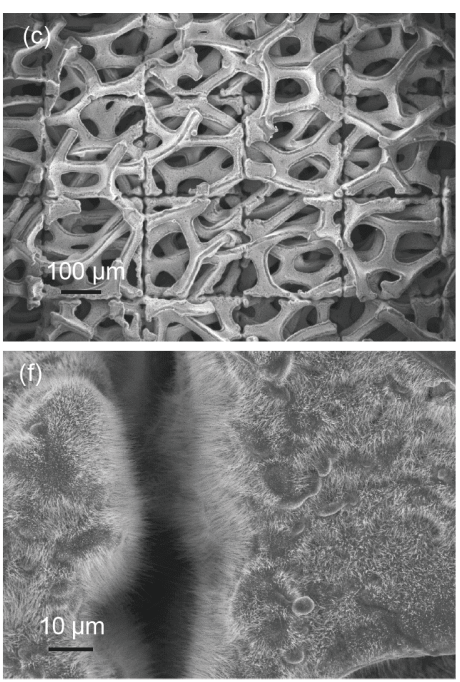

图 5 不同扫描间距下的氧化铜纳米线形貌图. (a), (d) $0.1 \mathrm{~mm}$; (b), (e) $0.3 \mathrm{~mm}$; (c), (f) $0.5 \mathrm{~mm}$

Figure 5 SEM images of the $\mathrm{CuO}$ nanowire surfaces with different scan spacings. (a), (d) $0.1 \mathrm{~mm}$; (b), (e) $0.3 \mathrm{~mm}$; (c), (f) $0.5 \mathrm{~mm}$. 
的纳米线长度偏短, 更稀疏, 激光烧蚀过的区域在热氧 化的过程中纳米线生长的密度较大, 长度基本在 4-10 $\mu \mathrm{m}$. 通过图4和 5 的分析表明, 激光织构的不同参 数对氧化铜纳米线的生长情况有一定的影响.

\section{2 样品表面元素分析}

采用EDS对原始样品组、激光预处理未氧化组以 及激光预处理后热氧化组三组样品表面的元素及其相 对质量百分比进行分析, 并对激光预处理后热氧化组 的激光扫描区域和未扫描区域分别进行测量. 测试结 果如表1所示, 可以看出样品表面均存在 $\mathrm{C}, \mathrm{Cu}, \mathrm{O}$ 三种 元素, 但各组样品的相对质量百分比存在差异. 原始样 品表面 $\mathrm{C}$ 的相对质量百分比为 $3.05 \%, \mathrm{Cu}$ 的相对质量百 分比为 $96.80 \%$, O仅有 $0.15 \%$, 说明铜为主要元素, 表面 存在极其轻微的氧化. 经过激光预处理未氧化的样品 表面 $\mathrm{O}$ 的相对质量百分比提高至 $14.88 \%$, 可见激光微 织构的过程提高了样品表面的氧化程度.

激光预处理后热氧化组表面 $\mathrm{O}$ 的相对质量百分比 都要大于其他两组, 结合表面形貌图可表明是 $\mathrm{CuO}$ NWs的生长. 此外, 在该组样品激光未扫描区域表面, $\mathrm{O}$ 的相对质量百分比为 $21.44 \%$, 而在激光扫描区域上 $\mathrm{O}$ 的相对质量百分比为 $32.68 \%$, 进一步说明激光织构对 泡沫铜表面氧化程度的提高主要表现在激光扫描路径 所经过的区域.

为进一步确定 $\mathrm{CuO} \mathrm{NWs}$ 的表面成分, 采用 X射线 衍射仪对样品进行物相分析. 图6(a)和(b)分别为原始

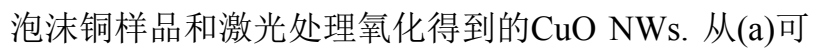
以看出, 分别在 $2 \theta=43.3^{\circ}, 50.5^{\circ}$ 和 $74.2^{\circ}$ 处出现衍射峰 (JCPDS:70-3039), 表明原始样品的表面主要存在铜单 质. 在(b)中, 分别在 $2 \theta=35.5^{\circ}, 38.7^{\circ}$ 和 $48.7^{\circ}$ 处出现的衍 射峰, 对应 $\mathrm{CuO}$ 的(-111), (111), (-202)晶面, 表明样品 表面存在 $\mathrm{CuO}$ (JCPDS:80-1916). 分别在 $2 \theta=29.6^{\circ}$, $36.4^{\circ}, 42.3^{\circ}, 61.4^{\circ}$ 和 $73.5^{\circ}$ 出现的衍射峰对应的是 $\mathrm{Cu}_{2} \mathrm{O}$ 的(110), (111), (200), (220)和(311)晶面衍射峰 (JCPDS:78-2076). XRD结果表明, 在热氧化法处理后, 样品表面存在 $\mathrm{CuO}$ 和 $\mathrm{Cu}_{2} \mathrm{O}$.

关于热氧化法生长氧化铜纳米线的生长机理, 被 广泛认可的是晶界扩散动力学模型 ${ }^{[22]}$. 基于晶界扩散 动力学模型, 在热氧化过程中, 泡沫铜由上而下会依 次形成 $\mathrm{CuO}$ 层和 $\mathrm{Cu}_{2} \mathrm{O}$ 层作为生长 $\mathrm{CuO} \mathrm{NWs}$ 的前驱体. 在 $\mathrm{CuO}$ 层和 $\mathrm{Cu}_{2} \mathrm{O}$ 层界面处, $\mathrm{CuO}$ 转化为 $\mathrm{Cu}_{2} \mathrm{O}$ 时引起体
表 1 三组样品表面的EDS谱图

Table 1 EDS patterns of three sets of sample surfaces

\begin{tabular}{ccccc}
\hline 元素 & $\begin{array}{c}\text { 原始 } \\
\text { 泡沫铜 }\end{array}$ & $\begin{array}{c}\text { 激光织构 } \\
\text { 未氧化 }\end{array}$ & $\begin{array}{c}\text { 激光未 } \\
\text { 扫描处 }\end{array}$ & $\begin{array}{c}\text { 激光 } \\
\text { 扫描处 }\end{array}$ \\
\hline $\mathrm{C}$ & $3.05 \%$ & $9.05 \%$ & $4.82 \%$ & $7.65 \%$ \\
$\mathrm{O}$ & $0.15 \%$ & $14.88 \%$ & $21.44 \%$ & $32.68 \%$ \\
$\mathrm{Cu}$ & $96.80 \%$ & $76.07 \%$ & $73.73 \%$ & $59.67 \%$ \\
\hline
\end{tabular}

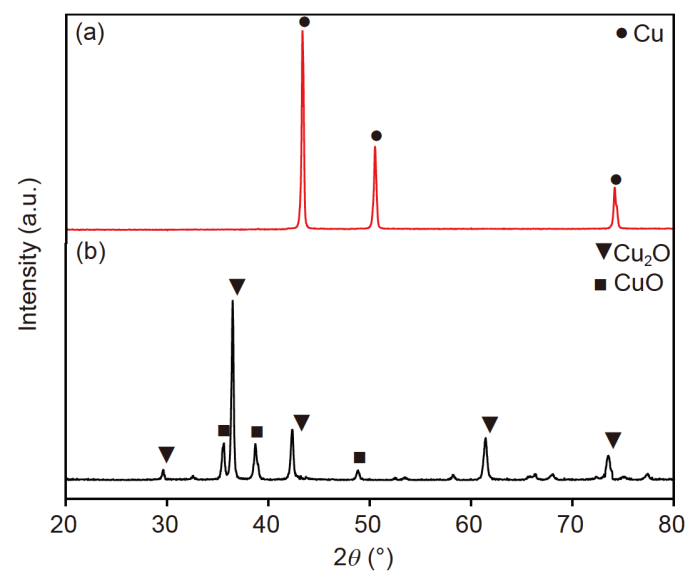

图 6 (网络版彩图)样品的XRD谱图. (a) 原始样品; (b) 氧化 铜纳米线(实心圆表示 $\mathrm{Cu}$; 三角形表示 $\mathrm{Cu}_{2} \mathrm{O}$; 方形表示 $\mathrm{CuO}$ ) Figure 6 (Color online) XRD patterns of (a) original copper foam; (b) $\mathrm{CuO}$ NWs film (solid circle: $\mathrm{Cu}$; triangle: $\mathrm{Cu}_{2} \mathrm{O}$; square: $\mathrm{CuO}$ ).

积收缩, 在该界面区域积累了压缩应力, 驱动铜离子扩 散至表面. 由于纳米线根部和尖部的铜离子浓度梯度, 通过与空气氧的反应将铜离子加入到纳米线的生长过 程中.

激光织构的过程烧蚀泡沫铜, 产生的熔融物被挤 出烧蚀区, 而后重新沉积于表面. 这些熔融物具有更 高的能量, 能够吸附更多的空气氧, 为热氧化过程提 供额外的 $\mathrm{CuO}$ 和 $\mathrm{Cu}_{2} \mathrm{O}$ 作为生长 $\mathrm{CuO} N W s$ 的前驱体, 扩 大了纳米线根部和尖部的铜离子浓度梯度, 从而改善 $\mathrm{CuO} \mathrm{NWs}$ 的生长情况. 未经过激光织构直接进行热氧 化生长的氧化铜纳米线, 铜与氧仅在原始泡沫铜表面 接触. 而激光织构在泡沫铜表面形成了周期性微结构, 其产生的微凹槽在一定程度上为铜与氧提供了额外的 接触面积. 此外, 激光织构形成周期性微结构, 有助于 热应力从微结构的微边缘处释放. 因此, 激光织构再经 过热氧化得到的 $\mathrm{CuO} N W s$ 基本都保持直立生长, 而直 接热氧化法得到的 $\mathrm{CuO} \mathrm{NWs}$ 多呈杂草状倒伏. 


\section{3 光催化实验结果分析}

为了研究 $\mathrm{CuO} \mathrm{NWs}$ 光催化降解有机分子的能力, 选择甲基橙代替有机废水作为降解原料.

甲基橙的催化降解率采用下式 ${ }^{[23]}$ 计算:

$\eta=\frac{C_{0}-C}{C_{0}} \times 100 \%=\frac{A_{0}-A}{A_{0}} \times 100 \%$.

降解速度可由一级动力学方程 ${ }^{[11]}$ 表示:

$-\ln \left(\frac{C_{0}}{C}\right)=K t$,

式中, $\eta$ 表示甲基橙的降解率; $C_{0}$ 表示甲基橙的初始浓 度, $C$ 表示降解一段时间后的浓度; $A_{0}$ 表示甲基橙初始 吸光度, $A$ 表示降解一段时间后甲基橙的吸光度; $K$ 为 降解反应的速率常数.

光催化反应机理如图7所示 ${ }^{[24]}$. 当紫外光照射在 样品表面时, 样品表面的 $\mathrm{CuO}$ 和 $\mathrm{Cu}_{2} \mathrm{O}$ 会产生空穴-电子 对. 空穴从 $\mathrm{CuO}$ 的价带转移至 $\mathrm{Cu}_{2} \mathrm{O}$ 的价带, 同时, 光生 电子从 $\mathrm{Cu}_{2} \mathrm{O}$ 的导带转移到 $\mathrm{CuO}$ 的导带. 随后, 空穴和 电子分别反应产生・ $\mathrm{OH}, \mathrm{O}_{2}{ }^{-\cdot}$ 等高活性物质. 这些物质 能在短时间内将有机物氧化分解成 $\mathrm{H}_{2} \mathrm{O}, \mathrm{CO}_{2}$ 等无机 物. 然而对氧化铜而言, 其本身存在较高的空穴-电子 复合率, 单靠自身降解有机物的效率极低. 而 $\mathrm{H}_{2} \mathrm{O}_{2}$ 能 够捕获电子, 有效地抑制空穴-电子的复合, 因此本实 验中均加入 $1 \mathrm{~mL} \mathrm{H}_{2} \mathrm{O}_{2}$ 使氧化铜纳米线可更好地发挥 光催化降解作用 ${ }^{[19]}$.

光催化降解甲基橙实验结果如图8所示. 由图8(a) 可知, 在反应前 $20 \mathrm{~min}$ 内, 三组样品都保持相近的降解 速率, 随着时间变化, 经过激光预处理的 $\mathrm{CuO} \mathrm{NWs}$ 的 光催化降解速率明显加快. 在没有加催化剂的条件下,

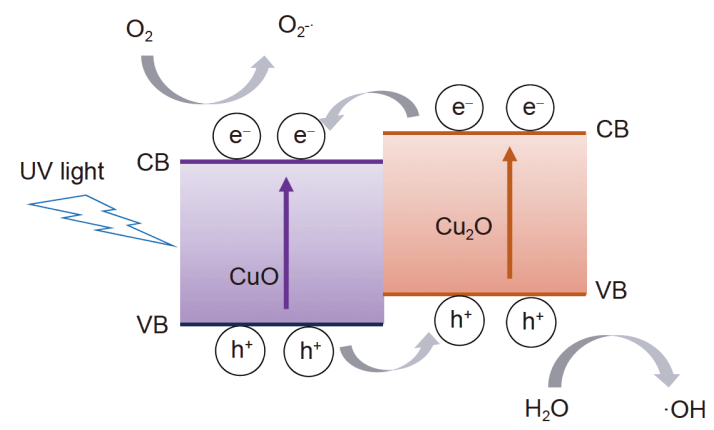

图 7 (网络版彩图)光催化降解甲基橙机理示意图

Figure 7 (Color online) Schematic illustration of photocatalytic degradation of methyl orange.
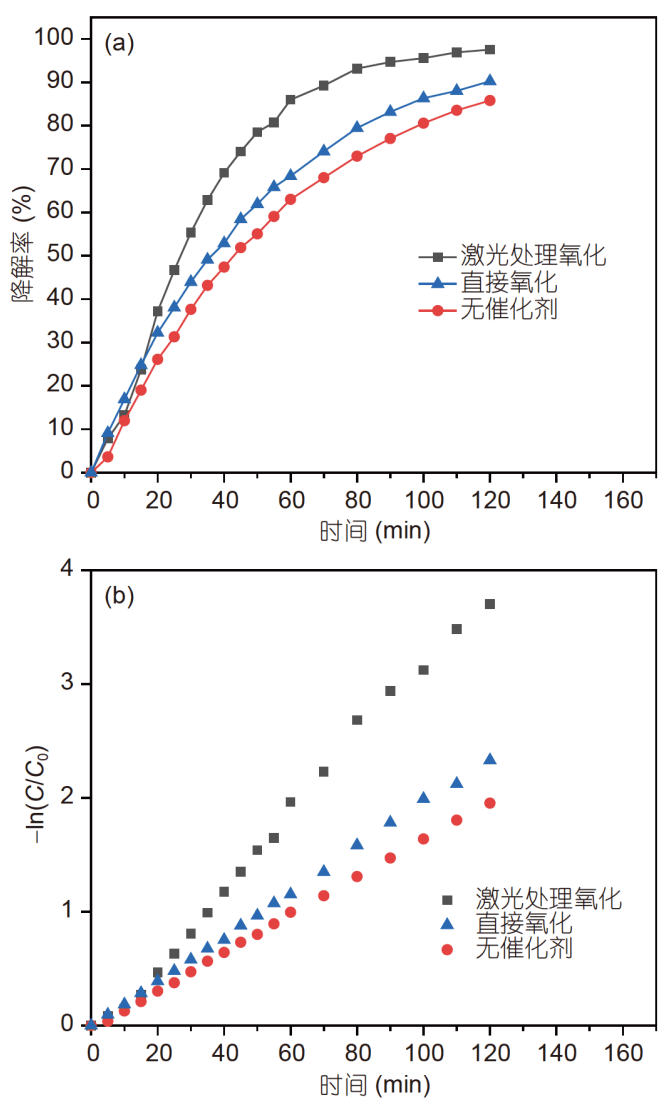

图 8 (网络版彩图)甲基橙随时间变化的降解情况. (a) 降解 率; (b) 降解速率(方形表示激光处理氧化组; 三角形表示直 接氧化组; 实心圆表示无催化剂组)

Figure 8 (Color online) Degradation of methyl orange (a) degradation rate; (b) degradation rate constant (square: $\mathrm{CuO}$ NWs prepared by laser texturing and thermal oxidation; triangle: $\mathrm{CuO}$ NWs prepared by direct thermal oxidation; solid circle: blank).

经过 $1 \mathrm{~h}$ 后甲基橙的降解率只达到 $63.1 \%$. 在加入直接 热氧化组的样品后，降解速率虽有提高但效果并不显 著, $1 \mathrm{~h}$ 后的降解率为 $68.4 \%$. 经过激光微织构的 $\mathrm{CuO}$ $\mathrm{NWs}$ 的降解率在 $1 \mathrm{~h}$ 后就达到 $87 \%$, 在 $2 \mathrm{~h}$ 后的降解率达 到 $97 \%$. 在图8(b)中, 经过激光织构后生长的 $\mathrm{CuO} \mathrm{NWs}$ 的降解速率常数为 $0.03305 \mathrm{~min}^{-1}$, 明显大于其他两组 的降解速率常数. 图9为经过光催化实验后的样品表 面形貌图, 其中 (a) 和(b)分别为激光处理氧化组和直接 氧化组. (a)中的纳米线经过实验后, 虽有部分弯折但 表面形貌未受影响, 基本无脱落情况; 而(b)中的纳米 线则几乎都脱落, 只剩根部.

结合表 1 的EDS数据可得, 激光织构后泡沫铜表面 覆有一定的熔融物使得 $\mathrm{O}$ 的相对原子质量百分比有明 显提高, 表明激光织构增加了样品表面 $\mathrm{CuO}$ 和 $\mathrm{Cu}_{2} \mathrm{O}$ 含 


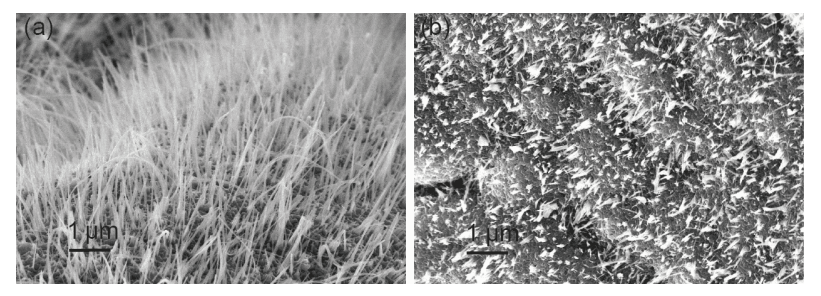

图 9 光催化实验后的样品表面形貌图. (a) 激光处理氧化 组; (b) 直接氧化组

Figure 9 SEM images of CuO NWs after photocatalytic experiment. (a) $\mathrm{CuO}$ NWs prepared by laser texturing and thermal oxidation; (b) $\mathrm{CuO}$ NWs prepared by direct thermal oxidation.

量, 不仅提供了更多用于光催化降解的反应物, 同时改 善了纳米线的生长情况, 使得 $\mathrm{CuO} \mathrm{NWs}$ 附着力好, 不 易脱落. 此外, 激光织构在泡沫铜表面形成了周期性微 结构, 其产生的微凹槽在一定程度上为铜与氧提供了 额外的接触面积, 促进了氧化铜纳米线长度和密度的 提高, 在一定程度上提高催化剂的比表面积. 因此, 经 过激光织构后热氧化生长的 $\mathrm{CuO} \mathrm{NWs}$ 展现出更好的 光催化性能.

为进一步验证激光微织构的氧化铜纳米线稳定 性, 进行了循环测试实验. 图10为氧化铜纳米线降解甲 基橙的循环实验结果. 由图 10 可看出，在经过 5 次的循 环降解实验后, 甲基橙的降解率并没有发生明显降低, 仍 可达到 $96 \%$. 实验结果进一步表明, 激光微织构对氧化铜 纳米线的光催化性能和稳定性提高有显著的促进作用.

\section{4 结论}

采用纳秒激光微织构与热氧化法相结合制备了泡

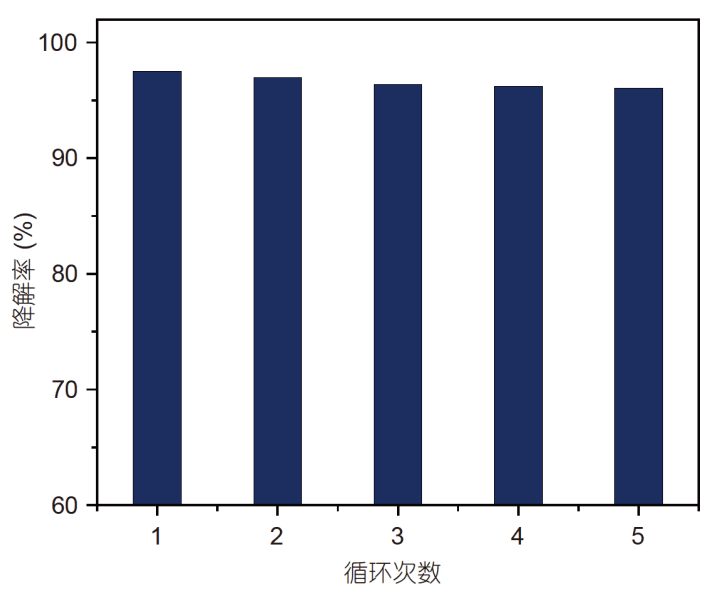

图 10 (网络版彩图)循环测试结果

Figure 10 (Color online) Degradation rate of methyl orange after 5 times' recycling test.

沫铜基底自支撑的 CuO NWs. 激光织构泡沫铜表面的 微阵列结构增大了纳米线的生长面积, 进一步释放热 应力, 提高了 $\mathrm{CuO} \mathrm{NWs}$ 的附着力. 此外, 激光预处理后 的样品表面氧元素含量的增加, 有效促进 $\mathrm{CuO} \mathrm{NWs}$ 的 生长, 使其密度和长度都有显著提高. 在不同的加工参 数下, 激光织构对 $\mathrm{CuO} \mathrm{NWs}$ 的生长情况有一定影响. 光催化降解甲基橙的实验结果表明, 将激光织构与热 氧化法相结合制备得到的 $\mathrm{CuO} \mathrm{NWs}$ 光催化剂, 纳米线 不易脱落, 具有更大的比表面积及更好的降解效果, 降 解率可提高至 $97 \%$. 同时, 此方法制备的光催化剂具有 稳定性好、可重复利用等优点, 循环测试5次以后, $\mathrm{CuO} \mathrm{NWs}$ 的降解率仍可保持在 $96 \%$, 具有更优异的光 催化性能.

\section{参考文献}

1 Singh R L, Singh P K, Singh R P. Enzymatic decolorization and degradation of azo dyes-A review. Int Biodeterioration Biodegradation, 2015, 104: 21-31

2 Martínez-Huitle C A, Brillas E. Decontamination of wastewaters containing synthetic organic dyes by electrochemical methods: A general review. Appl Catal B-Environ, 2009, 87: 105-145

3 Zhang L, Hu P, Wang J, et al. Adsorption of methyl orange (MO) by Zr (IV)-immobilized cross-linked chitosan/bentonite composite. Int J Biol Macromol, 2015, 81: 818-827

4 Lin H, Zhang H, Hou L. Degradation of C.I. acid orange 7 in aqueous solution by a novel electro/ $\mathrm{Fe}_{3} \mathrm{O}_{4} / \mathrm{PDS}$ process. J Hazard Mater, 2014, 276 : $182-191$

5 Xu Y, Xia J L, Huang X. State-of-the-arts/anaerobic membrane reactor for wastewater treatment and its future development (in Chinese). Membrane Sci Tech, 2016, 36: 139-149 [许颖, 夏俊林, 黄霞. 厌氧膜生物反应器污水处理技术的研究现状与发展前景. 膜科学与技术, 2016, 36: 139-149] 
$6 \mathrm{He} \mathrm{G}$, Zhang J, $\mathrm{Hu}$ Y, et al. Dual-template synthesis of mesoporous $\mathrm{TiO}_{2}$ nanotubes with structure-enhanced functional photocatalytic performance. Appl Catal B-Environ, 2019, 250: 301-312

7 Pant B, Park M, Park S J. $\mathrm{MoS}_{2} / \mathrm{CdS} / \mathrm{TiO}_{2}$ ternary composite incorporated into carbon nanofibers for the removal of organic pollutants from water. Inorg Chem Commun, 2019, 102: 113-119

8 Qian T T, Qian L Y, Du Y J, et al. Photocatalytic treatment of dye wastewater by magnetic $\mathrm{CuS} / \gamma-\mathrm{Fe}_{2} \mathrm{O}_{3}$ composites (in Chinese). Environ Protect Chem Ind, 2019, 39: 310-314 [钱婷婷, 钱露伊, 杜义佳, 等. 磁性 $\mathrm{CuS} / \gamma-\mathrm{Fe}_{2} \mathrm{O}_{3}$ 复合材料光催化处理染料废水. 化工环保, 2019, 39: 310-314]

$9 \mathrm{Wu} \mathrm{L}$, Yan $\mathrm{H}, \mathrm{Li} \mathrm{X}$, et al. Characterization and photocatalytic properties of $\mathrm{SnO}_{2}-\mathrm{TiO}_{2}$ nanocomposites prepared through gaseous detonation method. Ceram Int, 2017, 43: 1517-1521

10 Cheng S L, Chen M F. Fabrication, characterization, and kinetic study of vertical single-crystalline CuO nanowires on Si substrates. Nanoscale Res Lett, 2012, 7: 119

11 Scuderi V, Amiard G, Boninelli S, et al. Photocatalytic activity of $\mathrm{CuO}$ and $\mathrm{Cu}_{2} \mathrm{O}$ nanowires. Mater Sci Semiconductor Processing, 2016, 42: 89-93

12 Du X, Huang J, Feng Y, et al. Flower-like 3D CuO microsphere acting as photocatalytic water oxidation catalyst. Chin J Catal, 2016, 37: 123-134

13 Rao M P, Wu J J, Asiri A M, et al. Photocatalytic properties of hierarchical CuO nanosheets synthesized by a solution phase method. J Environ Sci, 2017, 69: 115-124

14 Carnes C L, Stipp J, Klabunde K J, et al. Synthesis, characterization, and adsorption studies of nanocrystalline copper oxide and nickel oxide. Langmuir, 2001, 18: 1352-1359

15 Neupane M P, Kim Y K, Park I S, et al. Temperature driven morphological changes of hydrothermally prepared copper oxide nanoparticles. Surf Interface Anal, 2009, 41: 259-263

16 Liu Q, Ding Y M, Li J W, et al. Research on the preparation of $\mathrm{CuO} / \mathrm{Al}_{2} \mathrm{O}_{3}$ and the mechanism of nitrobenzene degradation (in Chinese). Ind Water Treatment, 2018, 38: 15-20 [刘强, 丁一鸣, 李锦卫, 等. $\mathrm{CuO} / \mathrm{Al}_{2} \mathrm{O}_{3}$ 催化剂制备及降解硝基苯机理的研究. 工业水处理, 2018, 38: 1520]

17 Ren D D. Preparation of Nano-copper Oxide Assembly by Co-template Method and Its Catalytic Performance (in Chinese). Dissertation for Master's Degree. Jining: Qufu Normal University, 2014. 1-67 [任丹丹. 共模板法制备纳米氧化铜组装体及催化性能研究. 硕士学位论文. 济 宁: 曲阜师范大学, 2014. 1-67]

18 Jiang X, Herricks T, Xia Y. CuO nanowires can be synthesized by heating copper substrates in air. Nano Lett, 2002, 2: 1333-1338

$19 \mathrm{Yu}$ J J, Liao B, Zhang X, et al. Fabrication of $\mathrm{CuO}$ nanowires on copper foams by thermal oxidation and investigation of their photocatalytic roperties (in Chinese). Chin J Rare Metals, 2016, 40: 1021-1028 [于晶晶, 廖斌, 张旭, 等. 热氧化法在泡沫铜上制备CuO纳米线及其光催化性 能研究. 稀有金属, 2016, 40: 1021-1028]

20 Song Y, Wang C, Dong X, et al. Controllable superhydrophobic aluminum surfaces with tunable adhesion fabricated by femtosecond laser. Opt Laser Tech, 2018, 102: 25-31

21 Gregorčič P, Zupančič M, Golobič I. Scalable surface microstructuring by a fiber laser for controlled nucleate boiling performance of high- and low-surface-tension fluids. Sci Rep, 2018, 8: 7461

22 Yuan L, Wang Y, Mema R, et al. Driving force and growth mechanism for spontaneous oxide nanowire formation during the thermal oxidation of metals. Acta Mater, 2011, 59: 2491-2500

23 Zhou G H, Yang Z Y, Yang Y, et al. Photocatalytic degradation of organic dye with $\mathrm{H}_{4} \mathrm{SiW}_{12} \mathrm{O}_{40} / \mathrm{SiO}_{2}$ sensitized by $\mathrm{H}_{2} \mathrm{O}_{2}$ (in Chinese). Chin $\mathrm{J}$ Rare Metals, 2014, 38: 1073-1079 [周国辉, 杨志远, 杨谓, 等. $\mathrm{H}_{2} \mathrm{O}_{2}$ 处理 $\mathrm{H}_{4} \mathrm{SiW}_{12} \mathrm{O}_{40} / \mathrm{SiO}_{2}$ 光催化降解有机染料的研究. 稀有金属, 2014, 38: 10731079]

$24 \mathrm{Li} \mathrm{H}, \mathrm{Su} \mathrm{Z}, \mathrm{Hu} \mathrm{S}$, et al. Free-standing and flexible $\mathrm{Cu} / \mathrm{Cu}_{2} \mathrm{O} / \mathrm{CuO}$ heterojunction net: A novel material as cost-effective and easily recycled visible-light photocatalyst. Appl Catal B-Environ, 2017, 207: 134-142 


\title{
Fabrication and photocalytic performance of $\mathrm{CuO}$ nanowires on copper foam based on laser texturing
}

\author{
YAN HuangPing $^{1 *}, \mathrm{CHEN}_{\mathrm{ZiLu}}{ }^{1}, \mathrm{HE} \mathrm{Xin}^{1}{ }^{1}, \mathrm{ZHANG}_{\mathrm{KeX}}{ }^{1}$, SHEN SiHong ${ }^{1}$, \\ WANG ZhiGang ${ }^{2,3}$ \& ZHOU Rui ${ }^{1}$ \\ ${ }^{1}$ School of Aerospace Engineering, Xiamen University, Xiamen 361000, China; \\ ${ }^{2}$ Key Laboratory of Metallurgical Equipment and Control Technology, Ministry of Education, Wuhan University of Science and \\ Technology, Wuhan 430081, China; \\ ${ }^{3}$ Hubei Key Laboratory of Mechanical Transmission and Manufacturing Engineering, Wuhan University of Science and Technology, \\ Wuhan 430081, China
}

\begin{abstract}
Nanostructure $\mathrm{CuO}$ has attracted considerable research attention in the field of photocatalysis with excellent physical and chemical properties. However, most $\mathrm{CuO}$ photocatalytic nanomaterials prepared by chemical methods have problems such as low recycling rate and secondary pollution due to their morphological characteristics. In this paper, $\mathrm{CuO}$ nanowires (CuO NWs) grown based on copper foam were successfully prepared by laser texturing combined with subsequent thermal oxidation and used as photocatalyst for photocatalytic degradation of methyl orange (MO) solution. The effect of laser texturing parameters on the growth of copper oxide nanowires was investigated, such as scan spacing and scan times. After optimization, the appropriate laser processing parameters are chosen to form the micro-patterned grids on the copper foam. Subsequently, $\mathrm{CuO}$ NWs are synthesized by thermal oxidation. The structure morphology and chemical composition of samples were characterized by scanning electron microscopy (SEM), energy dispersive spectrometer (EDS) and X-ray diffraction (XRD). The photocatalytic performance of $\mathrm{CuO}$ nanowires based on copper foam was evaluated by degradation of methyl orange (MO) solution under ultraviolet light irradiation. The experimental results show that laser texturing of copper foam can not only increase the growth area of nanowires but also improve the content of thermally activated oxygen on the substrate. Therefore, the growth of $\mathrm{CuO} \mathrm{NWs} \mathrm{is} \mathrm{effectively} \mathrm{promoted} \mathrm{in}$ terms of length and density. The photocatalytic degradation rate of $\mathrm{MO}$ solution by $\mathrm{CuO} \mathrm{NWs}$ is up to $97 \%$ after $120 \mathrm{~min}$. After five cycles' measurement, the degradation rate can still remain 96\%. It demonstrates the good-performance stability and high recyclability of $\mathrm{CuO}$ NWs based on copper foam as photocatalysts. This study provides a feasible approach to synthesize $\mathrm{CuO} \mathrm{NWs}$ on the copper foam and effectively extend the potential application of $\mathrm{CuO}$ nanomaterials in the industrial wastewater treatment.
\end{abstract}

copper foam, $\mathrm{CuO}$ nanowires, laser texturing, photocatalytic degradation

PACS: $81.05 . \mathrm{Rm}$, 61.46.-w, 42.62.Cf, 81.16.Hc

doi: 10.1360/SSPMA-2019-0239 